\title{
Occurrence and risk assessment of multiclass endocrine disrupting compounds in an urban tropical river and a proposed risk management and monitoring framework
}

\begin{abstract}
Endocrine disrupting compounds (EDCs) are an emerging environmental concern and commonly occur as a mixture of compounds. The EDC mixture can be more toxic than any single compound. The present study analyses EDCs in surface water in the case of an urban tropical river, the Langat River, using the multiresidue analytical method of solid phase extraction and liquid chromatography-tandem mass spectrometry (SPE-LC-MS/MS). The Langat River is used as a drinking water source and is treated for Malaysian drinking water supply. A total of 14 EDCs i.e. five hormones, seven pharmaceuticals, one pesticide, and one plasticizer were detected. Caffeine was observed to be highest at $19.33 \mathrm{ng} / \mathrm{L}$, followed by bisphenol A and diclofenac at $8.24 \mathrm{ng} / \mathrm{L}$ and $6.15 \mathrm{ng} / \mathrm{L}$, respectively. Using a conservative risk quotient (RQ) method, EDCs were estimated for having negligible risks under acute and chronic exposure $(\mathrm{RQ}<0.002$ and RQmix $<0.003$; RQ $<0.01)$, suggesting that there is currently an insignificant ecological risk related to these compounds in the Langat River riverine ecosystem. However, the presence of EDCs in surface water raises concerns about potential human exposure to EDCs via dietary intake i.e. food and drinking water supply. Although the ecological risks are considered negligible, these risks should not be neglected in terms of future prioritization and risk management. Improvements in water quality monitoring and risk assessment in water source protection are required to support a multibarrier approach to managing drinking water supply systems for safe water supply. The present study proposes a risk management and monitoring framework for EDCs to support the aforementioned multibarrier approach.
\end{abstract}

Keyword: Emerging contaminants; Endocrine disrupting compounds (EDCs); Risk assessment; Riverine ecosystem; Surface water; Water quality 